\title{
Plantar fasciitis
}

\author{
Amit Dwivedi ${ }^{1, *}$, Ashok Kumar ${ }^{2}$ \\ ${ }^{\mathbf{1}}$ Assistant Professor, ${ }^{2}$ Professor, Dept. of Orthopedics, Santosh Medical College \& Hospital, Ghaziabad, Uttar Pradesh, India
}

Corresponding Author:

Email: dramitdwivedi@yahoo.in

\begin{abstract}
Heel pain, mostly caused by plantar fasciitis (PF), is a common complaint of many patients who requiring professional orthopedic care and are mostly suffering from chronic pain beneath their heels. Plantar fasciitis (PF) is also referred to as plantar heel pain, heel spur syndrome, or painful heel syndrome. It is an enthesopathy (an abnormality or injury at the site of attachment of a ligament or tendon to bone) of the origin of the plantar fascia at the medial tubercle of the calcaneus. It leads to inflammation of the thick tissue that creates the arch of the foot. Plantar fasciitis can be a nuisance to treat and, to date, we've had little high quality evidence to guide us. For some time we've noted the similarities between plantar fascial problems and tendinopathy. Back in 2006 Scott Wearing wrote an excellent paper on how the two structures shared similar pathology and similar response to load. The present article reviews studies done on the anatomy of plantar fasciitis and their histo-pathological features, factors associated with planter fasciitis, clinical features, imaging studies, differential diagnoses, and diverse treatment modalities for treatment of planter fasciitis, with special emphasis on non-surgical treatment. Anti-inflammatory agents, plantar stretching, and orthosis proved to have highest priority; corticosteroid injection, night splints and extracorporeal shock wave therapy were of next priority, in patients with planter fasciitis.
\end{abstract}

Keywords: Plantar fasciitis, Plantar heel pain, Risk factors, Imaging studies, Treatment, Rehabilitation.

\section{Introduction}

Heel pain is a common presenting complaint in the foot and ankle practice, and plantar fasciitis $(\mathrm{PF})$ is the most common cause of chronic pain beneath the heel in adults, making up $11-15 \%$ of the foot symptoms requiring professional care among adults It is estimated that 1 in 10 people will develop planter fasciitis during their life time planter fasciitis, which is more common in middle-aged obese females and young male athletes, has a higher incidence in the athletic population though not all suffering require medical treatment. In the literature, planter fasciitis has been described as painful heel syndrome, chronic plantar heel pain, heel spur syndrome, runner's heel, and calcaneal periostitis., ${ }^{1,2}$

Epidemiology, incidence and prevalence: It comprises $80 \%$ causes of chronic heel pain in majority of patients It thought to affects athletics alone, however, also observed in people with sedentary life style as well. It is affected, neither by age nor by gender.

Etiology / Risk Factors: Although the etiology is not clear, however, there are varieties of different data on proposed risk factors:

1. Excessive sudden weight load on the foot due to obesity or pregnancy. It have been observed that when the body mass index (BMI) $>25 \mathrm{~kg} / \mathrm{m} 2$, there is 2 -fold increased risk for planter fasciitis. ${ }^{3}$

2. Inflammatory arthritis such as rheumatoid arthritis (RA) and spondyloarthropathy

3. Diabetes Mellitus

4. Hypothyroidism

5. Osteoarthritis

Local causes that lead to repeated micro-trauma of the plantar fascia suggested as follow:

a. Mechanical imbalances of the foot problems are due to the foot itself or the use of shoes with poor arch support including flip-flops or soft soles. Foot and arch problems includes; pes planus (flat feet), pes cavus (high arches) and reduced dorsiflexion of the ankle (equinus gastrocnemius). Case-control studies reported the association between these mechanical risk factors increases the risk of plantar heel pain. ${ }^{4}$

b. Heel spur (exostosis) studies have been conflicting for the association between planter fasciitis and heel spur as it was observed in an X-ray of normal population $(85 \%$ in patients with symptoms of plantar fasciitis vs. $46 \%$ of controls).

c. Long-distance running, especially running downhill or on uneven surfaces.

d. Tight Achilles tendon, which is a common cause, prolongs flexion of the foot causes shortening of the plantar fascia. Flexion of the foot causes shortening of the plantar fascia. Therefore, when the individual stand, it stretches the plantar fascia and accelerates pain. ${ }^{5,6}$

e. Sudden changes in activities can easily place the tissue in repeated stress over a short period, such as a sudden jump, starting a new running program, or even changing of footwear.

f. Occupations requiring prolonged standing.

Clinical Features and Diagnosis: The diagnosis of planter fasciitis is usually clinical and rarely needs to be investigated further. The patient complains of pain in the medial side of the heel, most noticeable with initial steps after a period of inactivity and usually lessens with increasing level of activity during the day, but will tend to worsen toward the end of the day. Symptoms may become worse following prolonged weight 
bearing, and often precipitated by increase in weight bearing activities.

Symptom of planter fasciitis is described as 'firststep pain' or 'post-static dyskinesia', which is pain or irritation at the heel rising after a period of non-weight bearing or inactivity. Such as rising from bed in the morning, standing up after working at the desk for several hours, or driving the car for a long period.

In severe cases, any activity that stretches the fascia as walking barefoot, climbing stairs, even toe walking will provoke pain. Thus, relieve by rest may radiate from the central part of the heel pad or the medial tubercle of the calcaneum to the medial longitudinal arch of the foot. The key physical finding is local point tenderness along the medial tuberosity of the oscalcis.

The pain could be trigger upon dorsiflexion of the patients pedal phalanges, which further stretches the plantar fascia (windlass mechanism).

Imaging Studies: Imaging studies are typically not necessary for diagnosis of planter fasciitis. In the clinical management of chronic heel pain, diagnostic imaging can provide objective information. This information can be particularly useful in cases that do not respond to first-line interventions, or when considering more invasive treatments (e.g. corticosteroid injection).

1. Lateral radiograph of the ankle should be the first imaging study. It is a good modality for assessment of heel spur, thickness of plantar fascia, and the quality of fat pad. Stress fractures, unicameral bone cysts, and giant cell tumors are usually identified with plain radiography.

2. Ultrasound examination is operator-dependent, but it proves to be significant when the diagnosis is unclear. In the literature, normal thickness of the plantar fascia when measured in ultrasound varies in range (mean 2-3 mm). People with chronic heel pain are likely to have a thickened plantar fascia with associated fluid collection, and that thickness values $>4.0 \mathrm{~mm}$ are diagnostic of plantar fasciitis. ${ }^{7}$

3. Plantar fascia thickness values have also been used to measure the effect of treatments and there is a significant correlation between decreased plantar fascia thickness and improvement in symptoms. ${ }^{7-9}$

4. MRI can be used in questionable cases, which fail conservative management or are suspected of other causes of heel pain, such as tarsal tunnel syndrome, soft tissue and bone tumors, osteomyelitis, subtalar arthritis, and stress fracture.

Differential Diagnosis: Although planter fasciitis is the most common cause of chronic plantar heel pain, there are multiple differential diagnoses (Old healed calcaneum fracture, Achilles tendinitis \& Morton metarsalgia) most of which can be excluded following a comprehensive history and physical examination.
Treatment: The natural history of planter fasciitis is often self-limited. However, the typical resolution time is anywhere from 6 to 18 months and sometimes longer, which can lead to dissatisfaction of patient and physician. Most experts agree that early recognition and management of planter fasciitis leads to short course of treatment and greater chance of success with conservative therapies..$^{10-13}$

Numerous interventions have been described for treatment of planter fasciitis, which include: rest, heat, ice pack, non-steroidal anti-inflammatory drugs (NSAIDS), heel pads, magnetic insole, night splints, walking cast, taping, plantar and Achilles stretching, ultrasound, steroid injection, extra-corporeal shock wave therapy, platelet-rich plasma injection, pulsed radiofrequency electromagnetic field therapy, and surgery. Unfortunately, few high-quality randomized, controlled trials have been made to support these therapies. All in all, a trial of conservative treatment is generally advised before more invasive interventions are attempted.

Stretching: Stretching may be in calf or plantar region. Numerous authors have recommended that calf stretching should be one of the interventions used for patients with planter fasciitis. A calf stretch is performed with the patient stands with staggered legs facing toward a wall, with both hands stretched out. ${ }^{14}$

The dosage for calf stretching can be either three minutes at a time, three times a day or five 20-s intervals, twice daily, as both have the same effect. The continuity of the connective tissue between the Achilles tendon and the plantar fascia as well as the fact that decreased ankle dorsiflexion is a risk factor in the development of plantar fasciitis provides some justification for calf stretching.

DiGiovanni et al. were the first to publish that tissue specific plantar fascia-stretching exercise is more effective than calf stretching in a randomized clinical trial. Moreover, it seems that plantar fascia-stretching exercise is more effective than low dose shock wave therapy in acute phase of planter fasciitis. ${ }^{14}$

Night Splints: The design of night splinting is to keep the patient's ankle in a neutral position overnight, passively stretching the calf and plantar fascia during sleep. There is no difference between the various types of the night splints whose purpose is to allow the fascia to heal. There is moderate evidence that night splints are useful in improving symptoms of planter fasciitis, which are recommended to be used for 1-3 months and should be considered as an intervention for patients with symptoms greater than 6 months in duration.

Orthosis: The rationale for use of foot orthoses was to decrease abnormal foot pronation that was thought to cause increased stress on the plantar fascia, but the pain reduction mechanism obtained by the use of insoles would be mostly related to its supporting function of the longitudinal arch and not to the overload reduction over the plantar surface. There appears to be no 
difference between prefabricated or custom foot orthoses in the results of treatment which is strongly recommended to be used to provide short-term (3 months) reduction in pain and improvement in function. There is inconclusive evidence with regard the longterm (12 months) use of orthotic devices

Local Injection of Steroids: When more conservative management is unsuccessful, steroid injection is a preferred option. There is no gold standard regarding the types and doses of local injection of corticosteroids. It is recommended that steroid injection should be performed with precise determination of the location, which can be easily achieved by using ultrasonographic guidance. Generally, the medial approach is likely to be less painful than a direct plantar approach. Injecting deep to the plantar fascia ensures adequate spread of the steroid preparation and reduces the risk of fat pad atrophy.

Corticosteroid injection has been shown to significantly reduce plantar fascia thickness as early as two weeks and one month following treatment. Additionally, there is a significant correlation between decreased plantar fascia thickness and improvement in symptoms. Results of a Cochrane review show that corticosteroid injection therapy has short-term benefit compared to control, and the effectiveness of treatment is not maintained beyond six months. Complications of steroid injection are not common. Reported complications of palpation-guided steroid injection are plantar fascial rupture, fat pad atrophy, lateral plantar nerve injury secondary to injection, and calcaneal osteomyelitis. However, these complications have not been reported following ultrasonographic-guided injections.

Extra-corporeal Shock Wave Therapy: Extracorporeal shock wave therapy (ESWT) can be of high or low energy. It has been claimed that the deep tissue cavitation effect causes micro rupture of capillaries, leakage of chemical mediators, and promotion of neovascularization of the damaged tissue. It is usually applied under intravenous sedation with or without local infiltrative anesthesia. ESWT is indicated if there is failure of other conservative modalities such as stretching exercises, casting or night splinting, and symptoms lasting for more than 6 months. As this is a relatively safe procedure, it could be considered before any surgical treatment and may be preferable to try before local steroid injection. Bilateral cases can be treated under a single anesthetic and full weight bearing may be started immediately. Prior steroid injections of over three times appear to be a poor prognostic factor for good recovery following ESWT. This modality is contra-indicated in bleeding diatheses.

The outcome of ESWT is not dependent on the presence of calcaneal spur where it does not change the radiographic appearance of the spur.
Presence of calcaneal bone marrow edema on MRI has been found to be a good predictive indicator for a satisfactory clinical outcome following ESWT.

There are inconclusive data whether to use local steroids or ESWT. Perifascial edema seems to be a useful criterion to address the therapy to the high resolution ultrasonographic-guided steroid injection treatment, while in cases without edema, treatment could be address to ESWT Both local steroid injection and ESWT are proved to be effective in treatment of planter fasciitis, but as steroid injection is more cost effective and has more reproducible results regardless of machine or operator, it is preferred. However, ESWT should be considered prior to any surgical treatment for recalcitrant planter fasciitis. ${ }^{15}$

Autologous Platelet Rich Plasma (PRP): There is substantially growing enthusiasm for the use of growth factor containing harvested blood/ platelet concentrate which, unlike steroids, can stimulate the reparative process. Current studies have revealed that local injection of platelet rich plasma (PRP) provides significant relief of pain and improvement of function, and the results seems to be comparable, and sometimes superior to local steroid injection. However, available data are limited by quality and size of the study, as well as length of follow-up, and are currently insufficient to recommend this modality for routine clinical use.

Surgery: Recalcitrant cases where symptoms persist for more than 6-12 months, even after adequate conservative treatment are usually selected for surgery. Before surgery nerve conduction and electromyographic studies should be considered to determine if the posterior tibial nerve is compressed.

Open or endoscopic plantar fascia release may be done. Some advantages of endoscopic plantar fasciotomy include: minimal soft tissue dissection, excellent visualization of the plantar fascia, minimal postoperative pain, and earlier return to work, however, the American Orthopaedic Foot and Ankle Society recommends that in case of suspected nerve compression, endoscopic release should be avoided. All in all, still, the procedure of choice is open partial plantar fascia release with simultaneous release of first branch of lateral plantar nerve. A large cohort study indicates that $70 \%$ of patients showed improvement following surgery but only $50 \%$ of patients displayed complete satisfaction.

Following complete division of the plantar fascia, the development of pes planus, secondary hallux valgus, or hammer toes are expected, and therefore orthotics are required lifelong post-operatively.

\section{Conclusion}

Planter Fasciitis is the most common cause of inferior heel pain in adults. The patient usually complains of gradual onset of pain along the medial side of the heel. The pain is worse when arising in the morning which becomes less severe after the few steps. 
The diagnosis of planter fasciitis is usually clinical and rarely needs to be investigated by imaging or electromyographically.

In most patients with planter fasciitis, conservative treatment usually is sufficient. Initially, a period of rest accompanied by anti-inflammatory agents (ice pack/heat, NSAID's), stretching, and an orthosis are recommended. There is no difference in which types of orthosis is used, although plantar stretching seems to be more effective. If the patient remain symptomatic, corticosteroid injection and night splint (especially in patients with symptoms greater than 6 months in duration) may be reasonable. ESWT should be considered prior to any surgical intervention in patients with refractory planter fasciitis.

In a good majority of the patients, these modalities are sufficient and the patient will become symptom free.

However, if after 6-12 months of conservative treatment, the patient still has sufficient symptoms that interfere with their activities of daily life, surgical intervention should be considered. Moreover, newer treatment modalities such as local injection of platelet rich plasma (PRP) which may play more important roles in near future should also be considered.

\section{References}

1. Van Lunen B, Cortes N, Andrus T, Walker M, Pasquale M, Onate J. Immediate effects of a heel-pain orthosis and an augmented low-dye taping on plantar pressure and pain in subjects with plantar fasciitis. Clin J Sport Med 2011;21(6):474-9.

2. Riddle DL, Schappert SM. Volume of ambulatory care visits and patterns of care for patients diagnosed with plantar fasciitis: a national study of medical doctors. Foot Ankle Int 2004;25(5):303-10.

3. Frey C, Zamora J. The effects of obesity on orthopaedic foot and ankle pathology. Foot Ankle Int 2007;28(9):9969.
4. Buchbinder R. Clinical practice. Plantar fasciitis. $N$ Engl $J$ Med 2004;350(21):2159-66.

5. Porter D, Barrill E, Oneacre K, May BD. The effects of duration and frequency of Achilles tendon stretching on dorsiflexion and outcome in painful heel syndrome: a randomized, blinded, control study. Foot Ankle Int 2002;23:619-24.

6. Young CC, Rutherford DS, Neidfeldt MW. Treatment of plantar fasciitis. Am Fam Physician 2001;63(3):467-74.

7. Wearing SC, Smeathers JE, Sullivan PM, Yates B, Urry SR, Dubois P. Plantar fasciitis: Are pain and fascial thickness associated with arch shape and loading? Phys Ther 2007;87:1002-8.

8. Thomas JL, Christensen JC, Kravitz SR, Mendicino RW, Schuberth JM, Vanore JV, et al. The diagnosis and treatment of heel pain: a clinical practice guidelinerevision 2010. J Foot Ankle Surg 2010;49:S1-19.

9. Alvarez-Nemegyei J, Canoso JJ. Heel pain: diagnosis and treatment, step by step. Cleve Clin J Med 2006;73:46571.

10. Aklirat M, Sen C, Günes T. Ultrasonographic appearance of the plantar fasciitis. Clin Imaging 2003;27:353-7.

11. Stuber K, Kristmanson K. Conservative therapy for plantar fasciitis: a narrative review of randomized controlled trials. JCCA 2006;50:118-33.

12. Fabrikant JM, Park TS. Plantar fasciitis (fasciosis) treatment outcome study: Plantar fascia thickness measured by ultrasound and correlated with patient selfreported improvement. Foot (Edinb) 2011;21:79-14.

13. Gudeman SD, Eisele SA, Heidt RS, Jr, Colosimo AJ, Stroupe AL. Treatment of plantar fasciitis by iontophoresis of $0.4 \%$ dexamethasone. A randomized, double-blind, placebo-controlled study. Am J Sports Med 1997;25:312-6.

14. DiGiovanni BF, Nawoczenski DA, Lintal ME, Moore EA, Murray JC, Wilding GE, et al. Tissue-specific plantar fascia stretching exercise enhances outcomes in patients with chronic heel pain. A prospective, randomized study. J Bone Joint Surg Am 2003;85-A:1270-7.

15. Saber N, Diab H, Nassar W, Razaak HA. Ultrasound guided local steroid injection versus extracorporeal shockwave therapy in the treatment of plantar fasciitis. Alexandria J Med 2011;48:35-42. 